\title{
Carotid Intima Media Thickness in Obese Egyptian Children and Adolescent Zainab Al-Drawny ${ }^{1}$, Safaa Hamdy Ahmad Saleh', Ahmad Abdel Aziz El-Sammak ${ }^{2}$, Hisham Mahmoud Attia*1 \\ Departments of ${ }^{1}$ Pediatric and ${ }^{2}$ Diagnostic Radiology, Faculty of Medicine, Zagazig University, Egypt. \\ *Corresponding author: Hesham Mahmoud Attia, Telephone: \pm 201024683929, E mail: mosad8rashed@ gmail.com
}

\begin{abstract}
Background: Obesity is a significant public health crisis. Carotid artery Intima-Media Thickness (CIMT) is a new noninvasive ultrasound test that is being recommended to screen for heart disease in apparently healthy individuals. Objective: To determine the relationship between CIMT and obesity in children and adolescents.

Patients and Methods: This study was a cross-sectional study, which was done on 74 obese children aged 6-18 years. This study was done at the Pediatrics Department, Zagazig University Hospitals., during the period from 2017 to 2019. All children were subjected to history taking and clinical examination. Anthropometric measurements were measured. Carotid intima-media thickness quantification was estimated.

Results: The study resulted in 36 females (48.6\%) and 38 males (51.4\%). Children lived in rural areas 38(51.4\%) and urban areas were $36(48.6 \%)$. There was a significant increase in CIMT in obese children. Statistically significant positive correlations were observed between CIMT and BMI. Statistically significant positive correlations between CIMT and total cholesterol, triglycerides, while no statistically significant correlations were observed between CIMT and LDL, and HDL.

Conclusion: There is a relationship between CIMT and obesity in children of the target population of the study and, at the same time, there also exist statistically significant positive correlations between CIMT and total cholesterol, triglycerides, and no statistically significant correlations between CIMT and LDL, and HDL. Regarding the prevalence of insulin resistance (IR) among our studied children, we found that 31 patients were IR (41.9\%), while 43 were not $(58.1 \%)$. Insulin resistance (IR) was statistically higher in children with CIMT > 0.5 millimeters.
\end{abstract}

Key words: Carotid Intimamemdia Thickness, Obese, Children, Adolescents

\section{INTRODUCTION}

Obesity is an important public health crisis all over the world ${ }^{(\mathbf{1})}$. The prevalence of obesity is more than doubled between adults (rising from 15\% to 34\%), and also more than three times as in children and adolescents (rising from 5\% to 17\%) ${ }^{(2)}$. Childhood obesity is always associated with many diseases, especially cardiovascular diseases, diabetes mellitus type 2, obstructive sleep apnea, some types of cancer, and osteoarthritis. For that, obesity has been found to reduce life expectancy ${ }^{(\mathbf{3})}$.

Carotid artery Intimamedia Thickness (CIMT) is considered a new noninvasive ultrasound test which is recommended by the American Heart Association and the American College of Cardiology as has a role in screening for heart disease in all individuals ${ }^{(4)}$.

Touboul et al. (5) demonstrated that obese children present higher values in the CIMT and, at the same time, have more cardiovascular complications throughout their lives.

Schiel et al. ${ }^{\left({ }^{6}\right)}$ found a significant association between carotid IMT and weight, BMI, BMI-SDS, blood pressure, as well as various other metabolic parameters.

Abdel-Wahab et al. ${ }^{(7)}$ concluded that obesity in childhood and adolescents is associated with subclinical atherosclerosis.
Borda et al. ${ }^{(8)}$ determined the relationship between CIMT and obesity in children. They found a relationship between CIMT and obesity in children and a direct relation between CIMT and abdominal perimeter.

\section{AIM OF THE WORK}

This work aimed to determine the relationship between CIMT and obesity in children and adolescents.

\section{PATIENTS AND METHODS}

This study was a cross-sectional study, which was done on 74 obese children aged 6-18 years. This study was done at the Pediatrics Department, Zagazig University Hospitals., during the period from 2017 to 2019.

Inclusion criteria: Obese children aged 6-18 years were chosen (their body mass index which exceeds the age- gender-specific $95^{\text {th }}$ percentile.

Exclusion criteria: Obese children with genetic syndromes, Children receiving any medications like hormonal or corticosteroids, Children with endocrinal disorders as diabetes mellitus. Children with hypertension and Children with chronic illness and 
physical disability to exclude the effect of possible comorbidities of obesity.

Ethical approval and written informed consent: An approval of the study was obtained from Zagazig University academic and ethical committee.

Every patient signed an informed written consent for acceptance of the operation.

History taking included (Age, sex residence, and socioeconomic level).

\section{Anthropometric measurements included:}

Weight which was measured in light clothing without shoes and socks to the nearest $0.1 \mathrm{~kg}$ using a pre-calibrated body impedance analyzer. Height: was measured without shoes to the nearest $0.1 \mathrm{~cm}$ using a calibrated stadiometer (RAVEN EQUIPMENT LIMITED, ENGLAND). Body Mass Index (BMI): It equals weight in kilograms over height in meters square $\left(\mathrm{BMI}=\mathrm{Wt} / \mathrm{ht}^{2}\right)^{\left({ }^{(9)}\right.}$.

Waist Circumference: was measured at the minimum circumference between the iliac crest and the rib cage using a tape measure.

Carotid intima-media thickness quantification. was measured by B-mode ultrasound using a $10-\mathrm{MHz}$ linear transducer (Philips HD7).

The subjects were examined supine with the neck extended and the probe in the anterolateral position. All measurements of IMT were made in the longitudinal plane at the point of maximum thickness on the far wall of the common carotid artery along a $1 \mathrm{~cm}$ section of the artery proximal to the carotid bulb.

The IMT was defined as the distance between the intima-blood interface and the adventitia-media junction. After freezing the image, the measurements were made using electronic calipers. The maximal thicknesses of the intima-media width were measured to give three readings and the mean value was used for statistical purposes.

\section{Laboratory investigations:}

- Fasting blood glucose: Done by kits pushed from the biotechnica instrument, Roma-Italy, with LOT Number is 000566, using Biosystem AIA Autoanalyser.

- METHOD: Colorimetric enzymatic method GODPOD.

- Fasting blood insulin: Done by kits pushed from
Monobind, USA, its LOT Number is \#EIA58K1K5, using ELISA Best-2000 Reader. TEST PRINCIPLE: The Insulin Quantitative Test Kit is based on a solid phase enzyme-linked immunosorbent assay.

- Fasting blood Triglyceride: Done by kits pushed from the biotechnica instrument, Roma-Italy, with LOT number is 000276, using Biosystem AIA Autoanalyser.

- Cholesterol: Done by kits pushed from biotecnica instrument, Roma-Italy, with LOT number is 000116, using Biosystem AIA Autoanalyser.

- METHOD: Colorimetric enzymatic method CHOD-PAP.

- HDL Cholesterol: Done by kits pushed from AMS U.K. Ltd, United kingdom, with LOT number is A8635, using Biosystem AIA Autoanalyser.

- METHOD: IMMUNOINHIBITION.

- LDL Cholesterol: LDL-C is most often measured indirectly, using a calculation based on other blood lipid analytes. Friedewald calculation incorporates total cholesterol, HDL-C, and triglyceride :

- LDL-C $(\mathrm{mg} / \mathrm{dl})=$ total cholesterol - HDL-C (triglycerides/5).

- HOMA was calculated by multiplying the value of fasting insulin and fasting glucose and divided by 22.5. The score of $\geq 4.0$ was classified as insulin resistance, while a score of less than 4.0 was considered as insulin sensitive ${ }^{(\mathbf{1 0})}$.

HOMA-IR=

Glucose x Insulin / (Glucose in molar unit mmol/l) 22.5

\section{Statistical analysis}

Recorded data were analyzed using the statistical package for social sciences, version 20.0 (SPSS Inc., Chicago, Illinois, USA). Quantitative data were expressed as mean \pm standard deviation (SD). Qualitative data were expressed as frequency and percentage .The following tests were done: Independent-samples t-test of significance was used when comparing two means. Chi-square $\left(\mathrm{X}^{2}\right)$ test of significance was used to compare proportions between qualitative parameters. Pearson's correlation coefficient (r) test was used to assess the degree of association between two sets of variables. $\mathrm{P}$ values $<0.05$ were considered statistically significant. 


\section{RESULTS}

Table (1): Demographic data of the studied children (Number=74)

\begin{tabular}{|l|l|c|c|}
\hline \multicolumn{2}{|l|}{} & Range & Mean+SD \\
\hline \multicolumn{2}{|c|}{ Age (years) } & $8-15$ & $11.70 \pm 1.66$ \\
\hline \multirow{2}{*}{ Sex } & Male & NO. & \% \\
& Female & 38 & 51.4 \\
\multirow{2}{*}{ residence } & Urban & 36 & 48.6 \\
\hline \multirow{3}{*}{ socioeconomic } & Rural & 38 & 48.6 \\
& HIGH & 32 & 51.4 \\
& MILD & 38 & 43.2 \\
& LOW & 4 & 51.4 \\
\hline
\end{tabular}

This table shows demographic data of the studied sample, the mean of age was $11.7 \pm 1.66$ years. The male percentage was $(51.4 \%)$ and the female percentage was of the urban residence (48.6\%) (Table 1).

Table (2): Anthropometric measurements, fasting insulin, glucose, and lipid profile of the studied children (Number=74)

\begin{tabular}{|c|c|c|}
\hline Anthropometric measurements & Range & Mean_SD \\
\hline Weight (kg) & $48-85$ & $67.46 \pm 8.47$ \\
\hline Height (cm) & $117-160$ & $143.11 \pm 8.79$ \\
\hline Waist (cm) & $60-105$ & $88.41 \pm 9.75$ \\
\hline Hip (cm) & $50-103$ & $77.1 \pm 15.5$ \\
\hline BMI $\left(\mathrm{Kg} / \mathrm{m}^{2}\right)$ & $30.10-36.70$ & $32.03 \pm 1.88$ \\
\hline Z.score (SD) & $0.80-4.80$ & $2.14 \pm 1.13$ \\
\hline waist/hip & $0.70-6.40$ & $1.86 \pm 1.17$ \\
\hline Fasting insulin $(\mu / \mathrm{ml})$ & $2.80-21.50$ & $10.70 \pm 4$ \\
\hline Fasting glucose (mg/dl) & $64-88$ & $77.7 \pm 7$ \\
\hline Serum cholesterol (mg/dl) & $118-227$ & $174.2 \pm 28.2$ \\
\hline Serum triglyceride (mg/dl) & $27-220$ & $114.30 \pm 40.765$ \\
\hline HDL (mg/dl) & $30-73$ & $48.62+9.44$ \\
\hline LDL (mg/dl) & $52-160$ & $103.75+28.79$ \\
\hline
\end{tabular}

This table shows that, Weight values ranged between 48 and 85 with a mean of $67.46 \pm 8.47$, Height $(\mathrm{cm})$ values had mean of $143.11 \pm 8.79$, BMI $\left(\mathrm{Kg} / \mathrm{m}^{2}\right)$ values ranged between 30.10 and 36.70 with a mean of $32.03 \pm 1.88$, Z. score (SD) values ranged between 0.80 and 4.80 with a mean of $2.14 \pm 1.13$, waist/hip values ranged between 0.70 and 6.40 with a mean of $1.86 \pm 1.17$.

Fasting insulin $(\mu / \mathrm{ml})$ of the studied children ranged between 2.80 and 21.50 with a mean of $10.70 \pm 4$ Mean value of fasting glucose $(\mathrm{mg} / \mathrm{dl})$ was 77.7. Serum cholesterol $(\mathrm{mg} / \mathrm{dl})$ values ranged between 118 and 227 with a mean of $174.2 \pm 28.2$, Serum triglyceride $(\mathrm{mg} / \mathrm{dl})$ values ranged between 27 and 220 with a mean of 114.30 \pm 40.765 , HDL $(\mathrm{mg} / \mathrm{dl})$ values ranged between 30 and 73 with a mean of $48.62 \pm 9.44$, LDL $(\mathrm{mg} / \mathrm{dl})$ values ranged between 52 and 160 with a mean of $103.75 \pm 28.79$ (Table 2).

Table (3): Prevalence of IR among the studied children.

\begin{tabular}{|l|l|c|c|}
\hline \multicolumn{2}{|l|}{ Variable } & No. $(\mathbf{N}=\mathbf{7 4})$ & \% (100\%) \\
\hline \multirow{2}{*}{$\begin{array}{l}\text { Insulin } \\
\text { resistance }\end{array}$} & Non-IR & 43 & 58.1 \\
\cline { 2 - 4 } & IR & 31 & 41.9 \\
\hline
\end{tabular}

This table shows that the Prevalence of IR among the studied sample was (41.9\%) (Table 3).

Table (4): Descriptive statistics of the studied children regarding CIMT (millimeter).

\begin{tabular}{|l|c|c|c|}
\hline & Range & Mean \pm SD & Median \\
\hline Right CIMT (millimeter) & $0.11-0.7$ & $0.42 \pm 0.16$ & 0.4 \\
\hline Left CIMT (millimeter) & $0.12-0.7$ & $0.43 \pm 0.15$ & 0.4 \\
\hline Average CIMT (millimeter) & $0.1-0.7$ & $0.42 \pm 0.16$ & 0.4 \\
\hline
\end{tabular}

This table shows that CIMT (millimeter), ranged between 0.1 and 0.7 with a mean of $0.42 \pm 0.16$ (Table 4). 
Table (5): Correlation between CIMT and other data.

\begin{tabular}{|l|c|c|}
\hline \multirow{2}{*}{ CIMT } & \multicolumn{2}{|c|}{ Pearson's correlation } \\
\cline { 2 - 3 } & $\mathbf{r}$ & $\mathbf{p}$ \\
\hline Age (year) & $0.152+$ & 0.196 \\
\hline Weight $(\mathbf{c m})$ & $0.142+$ & 0.228 \\
\hline Height $(\mathbf{c m})$ & $0.154+$ & 0.189 \\
\hline Waist $(\mathbf{c m})$ & $0.012+$ & 0.921 \\
\hline Hip $(\mathbf{c m})$ & $-0.043-$ & 0.716 \\
\hline BMI $\left(\mathbf{K g} / \mathbf{m}^{\mathbf{2}}\right)$ & $0.249+$ & $0.032^{*}$ \\
\hline Z.score $(\mathbf{S D})$ & $0.356+$ & $0.002^{* *}$ \\
\hline Waist/hip $(\boldsymbol{\mu} / \mathbf{m l})$ & $-0.045-$ & 0.702 \\
\hline F insulin $(\mathbf{m g} / \mathbf{d l})$ & $-0.205-$ & 0.080 \\
\hline Serum cholsterol (mg/dl) & $0.368+$ & $0.001^{* *}$ \\
\hline Serum triglyceride (mg/dl) & $0.313+$ & $0.007 * *$ \\
\hline HDL (mg/dl) & $-0.148-$ & 0.207 \\
\hline LDL (mg/dl) & $-0.073-$ & 0.539 \\
\hline
\end{tabular}

There were statistically significant positive correlations between CIMT and (BMI, Z.score, serum cholesterol, and serum triglyceride). There were no statistically significant correlations between CIMT and other variables (Table 5).

Table (6): Frequencies of CIMT (millimeter) among the studied subjects.

\begin{tabular}{|c|c|c|c|}
\hline \multirow{2}{*}{ CIMT categories } & $<\mathbf{0 . 5}$ & No. & \% \\
\cline { 2 - 4 } & $>\mathbf{0 . 5}$ & 62 & $83.8 \%$ \\
\hline
\end{tabular}

Frequencies of CIMT (millimeter) among the studied subjects, $<0.5$ were $62(83.8 \%)$ and $>0.5$ were $12(16.2 \%)$ (Table 6).

\section{DISCUSSION}

Our study included 36 female $(48.6 \%)$ and 38 male $(51.4 \%)$. Jagadesan et al. ${ }^{(11)}$ found that the prevalence of obesity was higher in girls than boys. Shabana and Vijay ${ }^{(12)}$ found the prevalence of obesity was higher in girls than boys.

Our study showed that children who lived in rural areas were $38(51.4 \%)$ and in urban areas were 36 (48.6\%). In a study among 340 female adolescents living in Cairo and surrounding rural areas, 35 percent of the girls were overweight and 13 percent were obese. Overweight was more prevalent in urban girls than in rural girls and more prevalent in girls with a higher socioeconomic standard than in those with lower socioeconomic status ${ }^{(13)}$. This growing trend can be due to improved accessibility and affordability of both junk foods and motorized transport technologies leading to greater ingestion of energy-dense foods, combined with reduced physical activity in the lower-income community ${ }^{(\mathbf{1 4})}$.

The results of this study revealed that there was a significant increase in CIMT in obese children. Kandil et al. ${ }^{(15)}$ found higher values of CIMT was found in obese children compared to controls ( $\mathrm{p}<0.05)$. Arenas et al. ${ }^{\left({ }^{16)}\right.}$ found a significant difference in the CIMT between healthy and obese children diagnosed by BMI. In 2001 French investigators Tounian et al. (17) described an increase in arterial wall rigidity of common carotids in 48 obese children as compared to controls.
Our study showed that there were statistically significant positive correlations between CIMT and BMI, which is in agreement with other studies ${ }^{(\mathbf{1 8})}$. Exposure to cardiovascular risk factors (hypertension, hyperlipidemia) in obese children may induce changes in the arteries, thereby contributing to impaired endothelial function ${ }^{(\mathbf{1 9})}$.

Our study demonstrated statistically significant positive correlations of the CIMT with BMI. Higher CIMT was significantly associated with increased BMI. Obesity and CIMT may share common genetic factors (20).

This coincides with the results of previous investigators who demonstrated that a higher BMI was strongly associated with increased CIMT ${ }^{\left({ }^{\circ}\right.}$. On the other hand, Giannini et al. ${ }^{(21)}$ did not find such a relation.

Such an association of CIMT was not observed in overweight patients in a longitudinal follow-up study by Freedman $\boldsymbol{e t}$ al. ${ }^{(22)}$, who found that CIMT was not increased among overweight children, which was against the findings demonstrated by other investigators who observed impaired endothelial function in healthy overweight children compared to controls as assessed by BMI and expressed by increasing left CIMT ${ }^{(23)}$.

Our study demonstrated statistically significant positive correlations between CIMT and total cholesterol, triglycerides, and no statistically significant 
correlations between CIMT and LDL, and HDL.

This agrees with Martos et al. ${ }^{(24)}$ who described elevated values for CIMT in obese children and the relationship with alterations in their lipid profile, inadequate vasodilation mediated by flow, and a direct relationship with AP. These children became very high cardiovascular risk patients when associated with other risk factors such as arterial hypertension, low-grade inflammation, and sedentarism.

Elkiran et al. (25) also found significant differences in 64 obese children and 24 overweight children in comparison with healthy children in the CIMT and AP values with a positive correlation between CIMT and the AP and MBI values. These authors suggest that the AP is associated with cardiovascular complications and is a useful indicator of central obesity, so AP should be quantified in all epidemiological studies that include pediatric obese patients. These findings present some similarities with the results of this study. However, there exists the limitation of not having biochemical markers measured in the blood to establish relationships between CIMT and insulin resistance levels and low-grade inflammation.

Our study showed that WC was not correlated with IMT which agreed with Hacihamdioğlu et al. ${ }^{\text {(26) }}$ found that WC was not correlated with IMT.

When studying the prevalence of IR among our studied sample, we found that 31 patients were IR (41.9\%).

This agreed with Iwani $\boldsymbol{e t} \boldsymbol{a l} .{ }^{(\mathbf{1 0})}$ who aimed to investigate the usefulness of triglyceride to HDL-C ratio (TG: HDL-C) as insulin resistance (IR) marker for overweight and obese children. A total of 271 blood samples of obese and overweight children aged 9-16 years of whom $49 \%$ of children were found to have IR as defined by HOMA-IR $\geq 4.0$

This study showed that IR was statistically higher in children was CIMT $>0.5$ millimeters.

This agreed with Huang et al. ${ }^{(2)}$ who found, IR was statistically higher in children with a high level of CIMT.

Limitation of the study: small numbers of patients and lack of sufficient studies in the subjects of the research in children.

\section{CONCLUSION}

There is a relationship between CIMT and obesity in children of the target population of the study and, there are statistically significant positive correlations between CIMT and total cholesterol, triglycerides, and no statistically significant correlations between CIMT and LDL, and HDL Regarding Prevalence of insulin resistance (IR) among our studied children, we found that 31 patients were IR $(41.9 \%)$. IR was statistically higher in children was CIMT $>0.5$ millimeters.

\section{RECOMMENDATION}

Further studies on different age groups in different areas in Egypt are mandatory.

Conflict of interest: The authors declare no conflict of interest.

Funding sources: The authors have no funding to report

\section{REFERENCES}

1. World Health Organization(2013): Obesity: Classification of weight according to body mass index; Report of a WHO Consultation. World Heal Organ 2013.

https://www.who.int/nutrition/publications/obesity/W HO_TRS_894/en/

2. Huang K, Zou CC, Yang XZ et al. (2010): Carotid intima-media thickness and serum endothelial marker levels in obese children with metabolic syndrome. Arch Pediatr Adolesc Med., 164(9):846-51.

3. Haslam D, James W (2010): Obesity. Lancet, 366:1197- 1209.

4. Darabian S, Hormuz M, Latif MA et al. (2013): The Role of Carotid Intimal Thickness Testing and Risk Prediction for the Development of Coronary Atherosclerosis. Curr Atheroscler Rep., 15(3): 306-312.

5. Touboul PJ, Hernández-Hernández R, Küçükoğlu $S$ et al. (2007): Carotid artery intima media thickness ,plaque and Framingham cardiovascular score in Asia ,Africa/Middle East and Latin America: the PARCAALA study .Int J Cardiovasc Imaging, 23:557-567.

6. Schiel R, Beltschikow W, Radon S et al. (2007): Increased carotid intima-media thickness and associations with cardiovascular risk factors in obese and overweight children and adolescents. Eur J Med Res., 12:503-508.

7. Abdel-Wahab AM, Atwa HA, El-Eraky AZ et al. (2011): Subclinical atherosclerosis in obese adolescents with normal left ventricular function. Saudi Med., 32(9):919-924.

8. Borda WA, Badillo FL, Suárez JC et al. (2015): Carotid intima media thickness in obese children. Rev. Colomb. Radiol., 26(2):4185-91.

9. World Health Organization (2015): WHO Training course on child growth assessment. World Health Organization: Geneva. World Heal Organ. 2015; Available at http://www.who.int/childgrowth/training/en/

10. Iwani NA, Jalaludin MY, Zin RM et al. (2017): Triglyceride to HDL-C Ratio is Associated with Insulin Resistance in Overweight and Obese Children. Sci Rep., 7:40055.

11. Jagadesan $S$, Harish R, Miranda $P$ et al. (2014): Prevalence of overweight and obesity among school children and adolescents in Chennai. Indian Pediatr., 51(7):544-9.

12. Shabana $T$, Vijay V (2009): Impact of socioeconomic status on the prevalence of overweight and obesity among children and adolescents in urban India. Open Obes J., 1:9-14.

13. Jackson RT, Rashed M, Saad-Eldin R (2003): Rural- 
urban differences in weight, body image, and dieting behavior among adolescent Egyptian schoolgirls. Int $\mathbf{J}$ Food Sci Nutr., 54(1):1-11.

14. Mahshid D, Noori AD, Anwar TM (2005): Childhood obesity, prevalence, and prevention. Nutr J., 4:24-29.

15. Kandil ME, Anwar GM, Fatouh A et al. (2010): Relation between serum homocysteine and carotid intima-media thickness in obese Egyptian children. J Clin Basic Cardiol., 13:8-11.

16. Borda WA, Badillo FL, Suárez JC et al. (2015): Carotid Intima Media Thickness in Obese Children. Rev Colomb. Radiol., 26(2):4185-91.

17. Tounian P, Aggoun Y, Dubern B et al. (2001): Presence of increased stiffness of the common carotid artery and endothelial dysfunction in severely obese children: a prospective study. Lancet, 358:1400-4.

18. Giannini C, De Giorgis T, Scarinci A et al. (2009): Increased carotid intima-media thickness in prepubertal children with constitutional leanness and severe obesity: the speculative role of insulin sensitivity, oxidant status, and chronic inflammation. Eur J Endocrinol., 161:73-80.

19. Raitakari OT, Juonala M, Kähönen M et al. (2003): Cardiovascular risk factors in childhood and carotid artery intima-media thickness in adulthood: the Cardiovascular Risk in Young Finns Study. JAMA., 290:2277-2283.

20. Juo SH, Lin HF, Rundek T et al. (2004): Genetic and environmental contributions to carotid intima-media thickness and obesity phenotypes in the Northern Manhattan Family Study. Stroke, 35:2243-7.

21. Giannini C, de Giorgis T, Scarinci A et al. (2008): Obese related effects of inflammatory markers and insulin resistance on increased carotid intima-media thickness in pre-pubertal children. Atherosclerosis, 197:448-56.

22. Freedman DS, Dietz WH, Tang R et al. (2004): The relation of obesity throughout life to carotid intimamedia thickness in adulthood: the Bogalusa Heart Study. Int J Obes Relat Metab Disord., 28:159-66.

23. Woo KS, Chook P, Yu CW et al. (2004): Effects of diet and exercise on obesity-related vascular dysfunction in children. Circulation, 109:1981-6.

24. Martos $\mathrm{R}$, Valle $M$, Morales $\mathrm{R}$ et al. (2006): Hyperhomocysteinemia correlates with insulin resistance and low-grade systemic inflammation in obese prepubertal children. Metabolism, 55:72-7.

25. Elkiran O, Yilmaz E, Koc M (2013): The association between intima-media thickness, central obesity, and diastolic blood pressure in obese and overweight children: A cross-sectional school-based study. Int J Cardiol., 165:528-32.

26. Hacihamdioglu B, Okutan V, Yozgat Y et al. (2011): Abdominal obesity is an independent risk factor for increased carotid intima-media thickness in obese children. Turk J Pediatr., 53:48-54. 